\title{
Dynamic programming and automated segmentation of optical coherence tomography images of the neonatal subglottis: enabling efficient diagnostics to manage subglottic stenosis
}

Konrad M. Kozlowski

Giriraj K. Sharma

Jason J. Chen

Li Qi

Kathryn Osann

Joseph C. Jing

Gurpreet S. Ahuja

Andrew E. Heidari

Phil-Sang Chung

Sehwan Kim

Zhongping Chen

Brian J.-F. Wong 


\title{
Dynamic programming and automated segmentation of optical coherence tomography images of the neonatal subglottis: enabling efficient diagnostics to manage subglottic stenosis
}

\author{
Konrad M. Kozlowski, ${ }^{a}$ Giriraj K. Sharma, ${ }^{b}$ Jason J. Chen, ${ }^{a, c}$ Li Qi, ${ }^{d}$ Kathryn Osann, ${ }^{b, c}$ Joseph C. Jing, ${ }^{a, c}$ \\ Gurpreet S. Ahuja ${ }^{b, e}$ Andrew E. Heidari, ${ }^{a}$ Phil-Sang Chung, ${ }^{f}$ Sehwan Kim, ${ }^{\mathrm{f}, \mathrm{g}}$ Zhongping Chen, ${ }^{\mathrm{a}, \mathrm{c}}$ and \\ Brian J.-F. Wong ${ }^{\mathrm{a}, \mathrm{b}, \mathrm{c}, *}$ \\ aUniversity of California Irvine, Beckman Laser Institute, Irvine, California, United States \\ bUniversity of California Irvine, Department of Otolaryngology-Head and Neck Surgery, Orange, California, United States \\ 'University of California Irvine, Department of Biomedical Engineering, Irvine, California, United States \\ dSouthern Medical University, School of Biomedical Engineering, Guangzhou, China \\ eChildren's Hospital of Orange County, Orange, California, United States \\ 'Dankook University, Beckman Laser Institute Korea, Cheoan, Republic of Korea \\ 'Dankook University, School of Medicine, Department of Biomedical Engineering, Cheoan, Republic of Korea
}

\begin{abstract}
Subglottic stenosis (SGS) is a challenging disease to diagnose in neonates. Long-range optical coherence tomography (OCT) is an optical imaging modality that has been described to image the subglottis in intubated neonates. A major challenge associated with OCT imaging is the lack of an automated method for image analysis and micrometry of large volumes of data that are acquired with each airway scan (1 to $2 \mathrm{~Gb})$. We developed a tissue segmentation algorithm that identifies, measures, and conducts image analysis on tissue layers within the mucosa and submucosa and compared these automated tissue measurements with manual tracings. We noted small but statistically significant differences in thickness measurements of the mucosa and submucosa layers in the larynx $(p<0.001)$, subglottis $(p=0.015)$, and trachea $(p=0.012)$. The automated algorithm was also shown to be over 8 times faster than the manual approach. Moderate Pearson correlations were found between different tissue texture parameters and the patient's gestational age at birth, age in days, duration of intubation, and differences with age (mean age 17 days). Automated OCT data analysis is necessary in the diagnosis and monitoring of SGS, as it can provide vital information about the airway in real time and aid clinicians in making management decisions for intubated neonates. $\odot$ The Authors. Published by SPIE under a Creative Commons Attribution 4.0 Unported License. Distribution or reproduction of this work in whole or in part requires full attribution of the original publication, including its DOI. [DOI: 10.1117/1.JBO.24.9.096001]
\end{abstract}

Keywords: diagnostic imaging; intubation injury; neonate; optical coherence tomography; subglottic stenosis; texture analysis.

Paper 190139R received Apr. 30, 2019; accepted for publication Aug. 20, 2019; published online Sep. 6, 2019.

\section{Introduction}

Subglottic stenosis (SGS) in the neonatal population poses a significant diagnostic and management challenge. Injury to the delicate subglottic epithelium in neonates can result from a myriad of causative factors, including cyclical micromotion of the ventilation circuit, bacterial infection, biofilm formation, and direct interfacing of the endotracheal tube (ETT) with the subglottic mucosa. ${ }^{1}$ Following acute mucosal inflammation, edema and fibrosis can lead to SGS, which in some cases can be progressive and life threatening. SGS has a contemporary incidence between $0 \%$ and $2 \%$ per year in these patients, with the mean total charge in discharges from hospitalization being upward of $\$ 110,000 .^{2-4}$ While direct laryngoscopy and bronchoscopy remain the diagnostic gold standard for SGS, this procedure requires general anesthesia and poses considerable risk of airway compromise. ${ }^{5}$ Hence, there exists a critical need for a less invasive and practical means for neonatologists

*Address all correspondence to Brian J.-F. Wong, E-mail: bjwong@uci.edu Presented at the SPIE Photonics West BiOS Conference. San Francisco, California. January 27, 2018. and otolaryngologists to image the upper airway in intubated neonates.

Optical coherence tomography (OCT) is a high-speed, micrometer-resolution, cross-sectional diagnostic imaging modality that has been previously described to characterize changes in the subglottic mucosa following endotracheal intubation in animals and humans. ${ }^{6-8}$ Long-range OCT (LR-OCT) is an advanced adaptation of this technology, with an imaging range up to $20 \mathrm{~mm} \cdot{ }^{9,10}$ A practical limitation of OCT imaging is the massive volume of images recorded per airway scan (amounts to 1 to $2 \mathrm{~Gb}$ ). OCT image processing and objective analysis can also be challenging and highly time-consuming, precluding real-time assessment of the airway and clinical decision-making. ${ }^{11,12}$ This limitation has also been observed in intravascular OCT imaging, where each scan can generate up to 1000 individual images and requires hours of offline manual analysis. ${ }^{13}$ If OCT were adopted in the neonatal intensive care unit (NICU) for SGS, thousands of images per patient would need to be analyzed, reconstructed, visualized, and then reviewed by an expert. Like other diagnostic studies widely used as screening measures, such as pap smears or complete blood counts, OCT image analysis would require a large component of automation for broad adoption in clinical medicine. ${ }^{14,15}$ 
Texture analysis of tissue images is a viable and automated process that has been previously utilized in tissue identification, pathologic quantification, and cancer detection. ${ }^{16-18}$ Texture properties represent tissue changes within an image and can be evaluated via spatial distributions of pixel intensities. Texture analysis can be investigated through gray-level occurrence matrices (GLCM) or by statistical analysis of texture properties by examining the spatial distributions of pixels. ${ }^{17}$ In 2017, we described the dependence of two texture properties, correlation and homogeneity, on intubation time in a rabbit model of SGS. ${ }^{7}$ This was a first step in the long-term objective of automating analysis of thousands of OCT images per patient and optimizing airway management to avoid SGS in intubated neonates.

In this study, we examined two key issues relevant to the automated analysis of OCT images of the subglottis. First, we evaluated software aimed at segmenting the subglottic airway wall into two distinct layers and directly compared these results with manual segmentation. Second, to these segmented regions, we applied basic texture matrix analysis to quantitatively define tissue characteristics and compared this data to patient clinical parameters, such as gestational age (GA) or duration of intubation.

\section{Methods}

\subsection{Study Design}

Analysis was conducted on data acquired from a previous study of intubated neonates, which examined the use of OCT on the neonatal laryngotracheal airway. ${ }^{19}$ Each neonate was imaged in the NICU at either the University of California Irvine (UCI) or Children's Hospital of Orange County (CHOC). Clinical parameters including GA at birth and at time of imaging, total duration of intubation, ETT size, and patient weight were all recorded for each patient. Each subject's family provided written informed consent for participation, and imaging was performed following the protocol approved by the Institutional Review Boards at UCI and $\mathrm{CHOC}$.

\subsection{Optical Coherence Tomography System and Imaging}

Data sets for analysis were acquired through an LR-OCT system that has been previously described. ${ }^{19,20}$ In brief, a 1310-nm swept source laser with a repetition rate of $50 \mathrm{kHz}$ (Axsun Technologies, Massachusetts) was utilized as the light source of the OCT engine, and an acousto-optic modulator (Brimrose Corp., Massachusetts) was incorporated to generate a career frequency of $150 \mathrm{MHz}$ in the reference arm to achieve a 20-mm working distance. Flexible, side-view endoscopic OCT probes with an outer diameter of $0.7 \mathrm{~mm}$ and length of 65 to $70 \mathrm{~cm}$ were used for imaging the neonatal subglottis. A sterilized, distally sealed sheath encasing the probe was inserted into the subglottic region through the ETT in an intubated patient. To acquire images, the probe was rotated at 25 revolutions-per-second and linearly retracted at a speed of $1.56 \mathrm{~mm} / \mathrm{s}$. This continuous helical scanning scheme generated 300 to 600 360-deg images in total, with each image comprised of 2000 axial scans (A-lines) and each A-line storing 2048 12-bit pixels. Images were compressed into 8-bit bitmap for analysis.

\subsection{Data Collection and Selection}

Details of the methodology of OCT image acquisition have been previously described. ${ }^{19}$ Spiral OCT scans through the larynx, subglottis, and proximal trachea were obtained of each subject. A total of 58 OCT data sets were obtained, based on data from 49 different patients. Nine patients who were intubated for extended durations were serially imaged on different days. Each data set was evaluated for image quality prior to segmentation and texture analysis. Image quality was determined by the extent to which background noise affected the automated segmentation algorithm and the thickness measurements of the upper airway (mucosa and submucosa layers) that were produced. If the speckle noise or ghost images could not be initially cropped out of the OCT images or the noise significantly impacted the process of edge detection so that the anatomical structures of the image could not be accurately segmented [Figs. 1(e)-1(f)], the data set was excluded from analysis. Figures 1(a)-1(d) illustrate data with adequate image quality, and Figs. 1(e)-1(f) demonstrate examples of discarded data with image artifacts that precluded analysis. Many images had either minimal background noise [Figs. 1(a)-1(b)] or noise that was either filtered out of the image or did not significantly impact the process of segmentation [Figs. 1(c)-1(d)]. If the tissue contours of the mucosa and submucosa layers were faint or distorted, the data set was also excluded from analysis. In both circumstances, tissue segmentation by a human observer would also not be feasible.

Seventeen out of the 58 OCT data sets were excluded due to the aforementioned reasons. Out of the remaining 41 data sets, select trachea (3), subglottis (2), and larynx (2) subsets of data were discarded from within their respective data set for the same reasons. In those cases, the remaining analyzable images within the data set were included in the analysis. Each data set was divided into three airway segments, larynx, subglottis, and trachea, based on well-defined anatomic markers (e.g., true vocal fold margin, tracheal or cricoid cartilage, and laryngeal ventricles), which were identified in the OCT images. OCT has previously been used in the recognition of tissue structures. $^{21,22}$

Both automated and manual segmentations were performed by one trained study member (K.K.). Manual segmentation was performed on most cross-sectional images in 28 out of the total 41 data sets, whereas automated segmentation and texture analysis were performed on almost every one of the 41 data sets (38 trachea, 39 subglottis, and 39 larynx data sets). A single OCT data set ranged from 100 to 400 (mean 200) individual images, depending on how distal the OCT probe was positioned in the trachea at the beginning of the airway scan. The trachea typically included a greater number of frames compared to the larynx and subglottis, with a total count depending on how distal the OCT probe was positioned in the trachea at the beginning of the airway scan. Given the proportionally higher amount of trachea data, approximately every fifth tracheal image was segmented to achieve a proportionally sized data subset and to conserve time; at least 20 trachea images were analyzed per data set. In contrast, each and every image within the subglottis and larynx subsets was segmented; on average, there were approximately 40 data frames in each of these two airway segments. Approximately 100 total images were manually and autosegmented per data set. The amount of time required for the program to segment each data set was also recorded. 

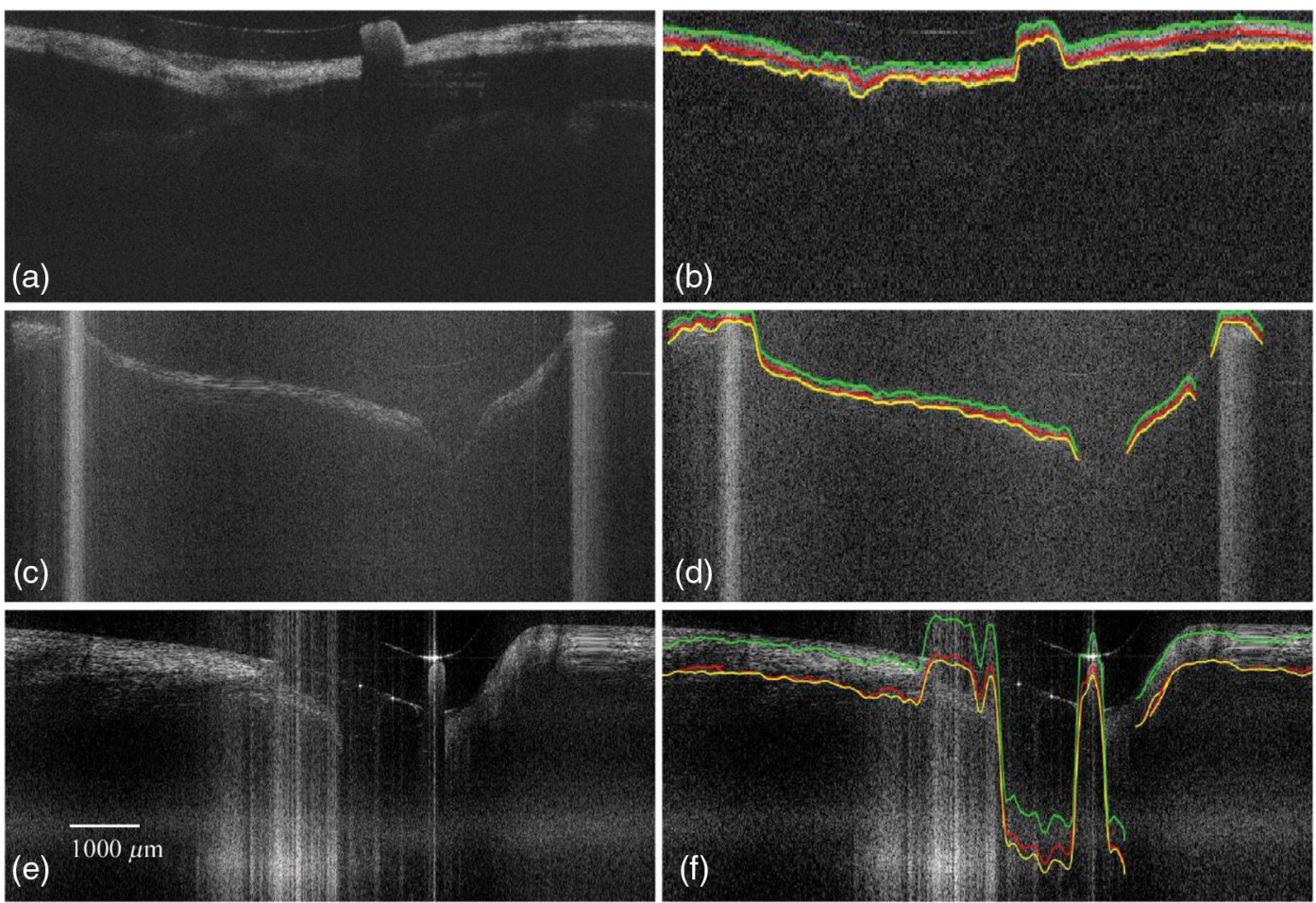

Fig. 1 (a), (c), (e) Axial LR-OCT images pre-segmentation and (b), (d), (f) post-autosegmentation. Images (a) and (c) are representative of high-quality data that was segmented for (b), (d) airway wall contours. Image (e) contained significant noise and artifact, (f) precluding segmentation.

\subsection{Manual Segmentation}

In manual segmentation, tracing of the airway wall layers was performed at user discretion with a computer mouse in a program written in MATLAB with graphical user interface. Anatomical OCT images of the airway are displayed in polar coordinates [Fig. 2(a)], though representation in Cartesian form facilitates better identification of key morphological features
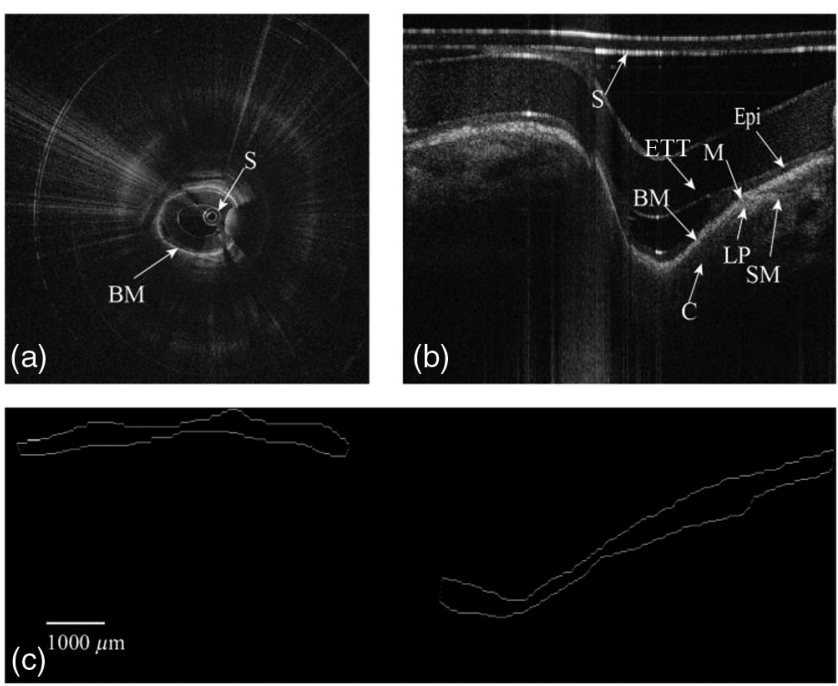

Fig. 2 Stepwise process of manual segmentation. Raw OCT data in (a) the polar coordinate and (b) Cartesian coordinate systems. (c) Tracing of airway-epithelium interface and basement membrane. $\mathrm{ETT}$, endotracheal tube; BM, basement membrane; C, cartilage; Epi, epithelium; S, OCT probe sheath; M, mucosa; SM, submucosa; $\mathrm{LP}$, lamina propria. during manual segmentation [Figs. 2(b) and 2(c)]. Layers of airway wall microstructure (e.g., epithelium, submucosa, and cartilage) were distinguished based on differences in grayscale (pixel) intensity, as each tissue layer has its unique optical composition. ${ }^{23}$ The first layer of the airway wall bordering the ETT is the mucosa, which consists of epithelium, basement membrane, and lamina propria. Underlying the mucosa is the submucosa, which is followed by cartilage tissue, when present. Two tissue interfaces were identified: the interface between the airway lumen and the mucosal epithelium, and the interface between the submucosa and cartilage. Both boundaries were traced and the mucosa-submucosa boundary was delineated by manual segmentation [Fig. 2(d)]. The layers identified were the same as those identified by autosegmentation, as described below.

\subsection{Automated Segmentation}

The automated segmentation algorithm that was developed for imaging bovine airway OCT images following smoke inhalation injury was adapted for use in this study. ${ }^{23}$ This graph theorybased segmentation algorithm was used on successive OCT images in each data set via a three-step process: a preprocessing step, followed by an edge detection algorithm, and lastly a thickness measurement heuristic (Fig. 3).

In the preprocessing step, the airway wall, including the mucosa and submucosa, was distinguished from the background (e.g., air, cartilage, ETT, OCT probe sheath, artifact, and noise). This step also addressed problems posed by speckle noise generated during imaging, ghost objects produced by internal interference of the optics, and mirror image/objects induced by Fourier transformation. ${ }^{24-26}$ These obstacles were compensated for with a series of low-level procedures, such as speckle noise 


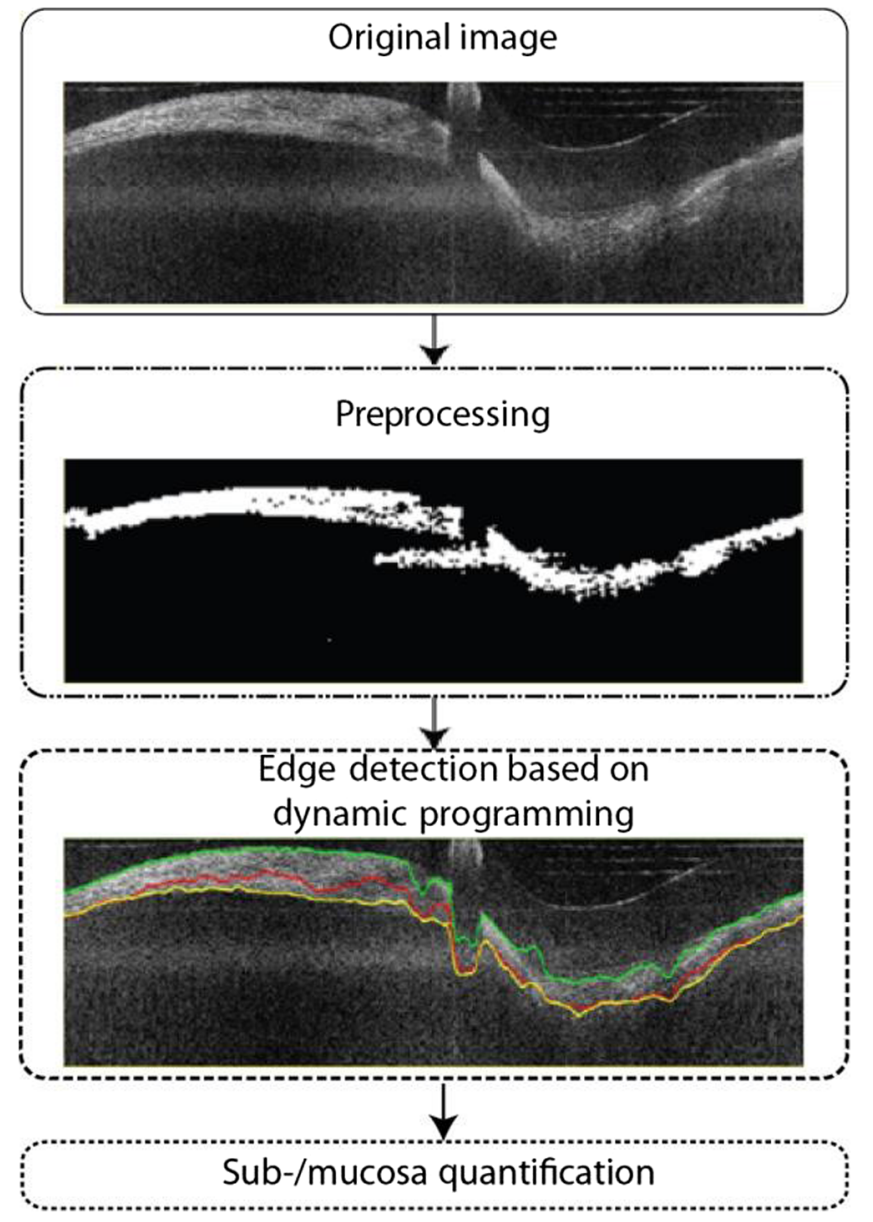

Fig. 3 Simplified three-step schematic diagram of the automated segmentation algorithm.

suppression, area filtering, dilation, or bridging. The different thresholds that controlled each of these procedures had to be manually set. Additionally, because the imaging probe was protected by a plastic sheath, it was necessary for this sheath to be identified and excluded from segmentation. Images were manually cropped to further eliminate any possible artifacts that could adversely affect the edge delineation step of the segmentation algorithm. Following frame cropping and threshold adjustment to suppress speckle noise, segmented grayscale images with reliable airway structures for edge detection were generated. These image stacks were subsequently rapidly analyzed by the program in real time on our computer workstation.

The edge detection step took previously generated localized airway regions and performed the segmentation task using a dynamic programming algorithm. ${ }^{23}$ The algorithm used graph construction and recursive solution finding to obtain the optimal path for the edge detection process. ${ }^{23}$ It treated every pixel in the OCT image as a node in a graph to find the shortest path (the edge) for the graph, based on the pixel intensity. The edge was refined, or smoothed, by averaging neighboring pixels. The airway lumen that was extracted was used as the first reference edge (epithelial surface) and the outer boundary of the airway wall, at the submucosa-cartilage junction represents the second reference edge. The middle edge, or the boundary between the mucosa and submucosa layers, was found by repeating this process in the closed region between the epithelium and cartilage edges. The average thickness of the mucosa and submucosa layers could then be determined after the mucosa and submucosa layers were accurately delineated.

\subsection{Texture Analysis}

Each autosegmented image was evaluated using texture analysis. $^{7,27,28}$ This was done using the GLCM-a statistical method of examining the spatial distributions of pixels. Four different tissue texture properties (correlation, homogeneity, contrast, and energy) were analyzed at four different angles of the GLCM (0 deg, $45 \mathrm{deg}, 90 \mathrm{deg}$, and $135 \mathrm{deg}$ ), resulting in 16 unique texture variables for each image. Texture analysis was performed on each frame in a data set in all 41 data sets. Automatic and manual segmentation measurements were compared using pairwise $t$-tests with significance level 0.05 . Associations between patient clinical parameters (e.g., GA at birth and duration of intubation) and texture variables were explored using Pearson correlations. As this was an exploratory analysis, significance levels were not adjusted for multiple comparisons.

\section{Results}

The automated and manual segmentation methods were found to have very small but statistically significant differences in thickness measurements. Sample sizes were 28, 27, and 23 data sets for the trachea, subglottis, and larynx, respectively (Table 1). Statistical analysis was performed after the segmentation of each data set, and airway segmentation plateaued with statistical significance around 28 data sets. Hence, manual segmentation was performed on only 28 of the 41 total data sets. We noted that the automated segmentation method tended to consistently but minimally underestimate the mean airway wall thickness (mucosa and submucosa) when compared to manual measurements, measured in pixels. Manual segmentation of each image stack ( $\sim 100$ frames) required 30 to $40 \mathrm{~min}$, depending on the total number of images, and this time was noted. Automated segmentation, compared to manual, was consistently faster $(p<0.01)$ for analysis of the trachea ( 9 times faster), subglottis (9 times faster), and larynx (6.7 times faster) (Fig. 4).

Analysis of the 16 different texture variables did not show any statistically significant associations with thickness measures but did demonstrate modest correlations with select patient variables. In the subglottis, there were moderate correlations between the contrast, energy, and homogeneity texture variables and the age in days of the neonate (Table 2). In addition, in the subglottis, the energy texture variables had a moderate correlation with the patient's GA at birth and the contrast texture

Table 1 Comparison between the airway wall thickness values (measured in pixels) obtained by automated and manual segmentations (paired $t$-test).

\begin{tabular}{lcccc} 
& \multicolumn{2}{c}{ Mean thickness } & & $P$-value \\
\cline { 2 - 3 } & Autosegmentation & Manual segmentation & \\
\hline Trachea & 38.013 & 38.852 & 0.012 \\
Subglottis & 44.793 & 46.657 & \\
Larynx & 52.183 & 55.069 & $<0.015$ \\
\hline
\end{tabular}




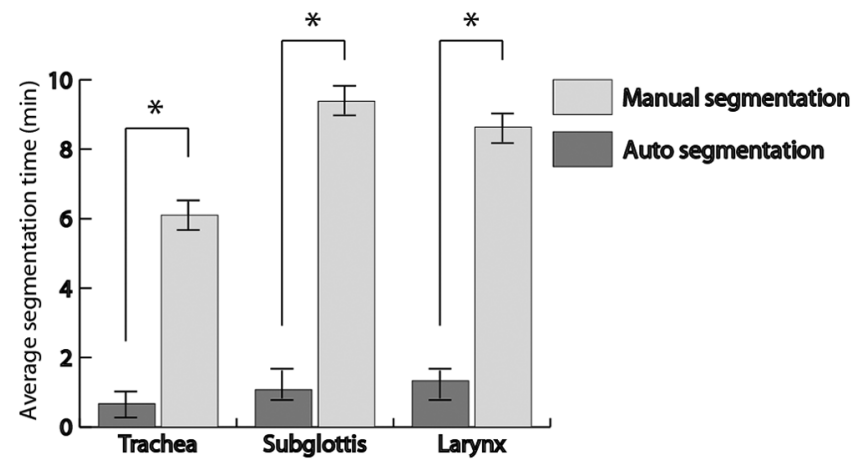

Fig. 4 Timewise comparison of manual and automated segmentations.

Table 2 Comparison between texture variables and clinical parameters in subglottis ( $t$-test).

\begin{tabular}{|c|c|c|c|}
\hline & Age in days & GA at birth & Days intubated \\
\hline Texture variables & $\begin{array}{c}\text { Correlation } \\
(p \text {-value })\end{array}$ & $\begin{array}{l}\text { Correlation } \\
\text { ( } p \text {-value) }\end{array}$ & $\begin{array}{l}\text { Correlation } \\
(p \text {-value })\end{array}$ \\
\hline Contrast (OD) & $0.317(0.049)$ & $-0.276(0.089)$ & $0.283(0.081)$ \\
\hline Contrast (45D) & $0.316(0.05)$ & $-0.285(0.079)$ & $0.282(0.082)$ \\
\hline Contrast (90D) & $0.333(0.039)$ & $-0.286(0.077)$ & $0.279(0.085)$ \\
\hline Contrast (135D) & $0.316(0.05)$ & $-0.285(0.079)$ & $0.282(0.082)$ \\
\hline Correlation (OD) & $0.184(0.262)$ & $-0.305(0.059)$ & $0.203(0.215)$ \\
\hline Correlation (45D) & $0.181(0.27)$ & $-0.28(0.084)$ & $0.207(0.206)$ \\
\hline Correlation (90D) & $0.25(0.125)$ & $-0.319(0.048)$ & $0.268(0.099)$ \\
\hline Correlation (135D) & $0.178(0.277)$ & $-0.28(0.084)$ & $0.203(0.216)$ \\
\hline Energy (OD) & $-0.347(0.031)$ & $0.33(0.04)$ & $-0.318(0.049)$ \\
\hline Energy (45D) & $-0.344(0.032)$ & $0.332(0.039)$ & $-0.315(0.05)$ \\
\hline Energy (90D) & $-0.347(0.031)$ & $0.332(0.039)$ & $-0.315(0.051)$ \\
\hline Energy (135D) & $-0.344(0.032)$ & $0.332(0.039)$ & $-0.315(0.051)$ \\
\hline Homogeneity (OD) & $-0.329(0.041)$ & $0.288(0.075)$ & $-0.293(0.07)$ \\
\hline Homogeneity (45D) & $-0.328(0.042)$ & $0.297(0.067)$ & $-0.293(0.071)$ \\
\hline Homogeneity (90D) & $-0.343(0.032)$ & $0.298(0.065)$ & $-0.29(0.074)$ \\
\hline $\begin{array}{l}\text { Homogeneity } \\
\text { (135D) }\end{array}$ & $-0.328(0.041)$ & $0.296(0.067)$ & $-0.293(0.07)$ \\
\hline
\end{tabular}

variables had a moderate correlation with the number of days the neonate was intubated (Table 2). Lastly, in the subglottis, there were consistent differences by age when categorized as below and above the median ( $\leq 17$ days versus $>17$ days) for the energy and homogeneity texture variables. Although these differences are consistent, due to the small sample size they would not reach statistical significance when adjusted for multiple comparisons (Table 3). Other clinical variables, including race, gender, or weight, showed no correlations with the texture variables.
Table 3 Comparison between texture variables and age of patient ( $\leq 17$ days versus $>17$ days) in subglottis $(t$-test).

\begin{tabular}{lc} 
& By Age $(\leq 17$ versus $>17)$ \\
\cline { 2 - 2 } Subglottis & Unadjusted $p$-value \\
\hline Energy (0D) & 0.025 \\
Homogeneity (0D) & 0.038 \\
Energy (45D) & 0.028 \\
Homogeneity (45D) & 0.044 \\
Energy (90D) & 0.026 \\
Homogeneity (90D) & 0.037 \\
Energy (135D) & 0.028 \\
Homogeneity (135D) & 0.044 \\
\hline
\end{tabular}

\section{Discussion}

OCT is a high-resolution cross-sectional imaging modality that is widely used in ophthalmologic, coronary vasculature, and dermatologic imaging. ${ }^{29-31}$ While OCT has been shown to reliably measure and characterize airway wall morphometry, it has limited practical applicability to airway monitoring due to the vast amount of data acquired with each scan. ${ }^{32-35}$ We aimed to address this need by constructing automated OCT data analysis methods, with the goal to ultimately provide clinicians with objective OCT data in a timely and practical manner.

\subsection{Automated Segmentation}

Unlike a manual approach, the autosegmentation code may erroneously classify noise in the OCT images as tissue structure, as it does not remove all noise during the preprocessing step of the algorithm. This occurs commonly with speckle noise at the lateral margins of the image and if it is not addressed, can lead to unreliable measurements during segmentation, resulting in an overestimation of wall thickness. To compensate, images must be analyzed with the selection of a specific and usually smaller than normal window size to find regions of pixel density that do not include speckle noise at the lateral margins of the image. The criteria used here thus tend to minimally but consistently underestimate the thickness of the mucosa and submucosa layers of the upper airway of patient data sets. More importantly, this minimal error comes with a critical gain in the consistency of autosegmentation program, as evident by our findings illustrated in Table 1.

Due to the necessity of the image preprocessing steps, the autosegmentation process was indeed semiautomated as opposed to fully automated, as some level of screening had to be performed. However, once the preprocessing steps were completed, the image stack was rapidly analyzed. More importantly, this entire semiautomated process, which encompassed the manual preprocessing and automated segmentation steps, was still significantly faster than a purely manual approach.

There is great clinical value in the rapid detection and segmentation of the upper airway wall. Edema can develop very quickly in the airway wall, and the state of the mucosa can change day by day. Therefore, clinicians need real-time data 
to determine whether to, for example, extubate or perform a tracheostomy on a patient, as other extubation readiness tests are of low value, and this type of real-time data can be obtained much more feasibly with an automated method of data analysis. ${ }^{36}$ Other studies have previously reported automated measurement of images, including that of CT and MRI images. ${ }^{37-39}$ However, OCT images have a much higher resolution and clarity compared to CT and MRI images, and this combined with our segmentation algorithm and texture analysis separates our image analysis from other studies.

\subsection{Texture Analysis}

Texture properties serve as an objective proxy for microscopic changes within native airway wall tissue. ${ }^{40,41}$ The optical properties of the airway wall differ between patients, presumably because airway wall microanatomy changes with physiologic states such as edema and fibrosis. Previous OCT studies have described texture analysis for the purpose of tissue classification. ${ }^{18,42-44}$ However, the correlation of texture properties with specific physiologic changes is not well understood. Ajose-Popoola et al. ${ }^{7}$ described texture analysis following OCT imaging of the rabbit subglottis following controlled brush injuries, in which significant correlations were noted between texture variables homogeneity and correlation with time from injury. These animal studies provided the motivation for the current analysis of in vivo human data sets.

We used Pearson correlations to identify associations between the texture variables and clinical neonate variables, such as GA at birth, intubation time, and ETT size. We noted moderate correlations between subglottic texture properties and GA at birth, age, and duration of intubation. Second, texture analysis of the subglottis showed consistent differences with age (median age of 17 days) at or near the significance level of $p<0.05$ before adjustment for multiple comparisons. Findings here suggest that texture analysis of OCT images may potentially be used to correlate physiologic changes in tissue composition with these specific patient metrics. For example, two neonates with respective intubation durations of 3 days and 3 weeks may indeed have similar subglottic wall thicknesses. However, the optical microstructure of their respective airway walls can be characterized by texture properties, which may help differentiate healthy tissue from edema in recently intubated neonates and fibrosis following lengthy or repeated intubations.

\subsection{Study Limitations}

As all imaging was performed in vivo, direct comparison of airway wall measurements and microanatomic composition with tissue histology was not possible, as laryngeal biopsy is generally never indicated for SGS management and postmortem evaluation of the neonate is not widely practiced as the emotional burden for families is often insurmountable. However, given prior animal OCT studies that demonstrate correlation of OCT and histology, our study offers a means to attain objective, structural information on the human subglottic airway. ${ }^{45}$

Texture analysis is a simple feature extraction technique that can be used as a reliable deterministic statistical process. Although it has its limitations, as various factors can affect the reliability, reproducibility, and robustness of texture features, we were able to use this rudimentary feature classification technology to show moderate correlations nearing significance between clinical neonate variables and certain texture variables, even with a small patient sample. ${ }^{46}$ While we obtained small effect sizes with this small patient sample, this is an early result that warrants further study. A larger sample size and future improvements in the segmentation algorithms may lead to stronger associations between clinical neonate variables and texture variables, resulting in a larger effect size. Although texture analysis is a well-established albeit rudimentary pattern recognition approach, other pattern recognition and feature extractions methods, such as fuzzy clustering, could also be also used to find evidence of changes in tissue structure. ${ }^{47}$ Fuzzy clustering is a process that sorts specific elements of tissue into different classes so that elements in a class are similar to each other. It has already been used to analyze nerve fibers in glaucoma patients as well as to evaluate breast cancer nuclei as malign or benign and advance breast cancer diagnosis. ${ }^{48,49}$ As OCT is a high data volume imaging modality with multispecialty applications, this underscores the critical need to develop automated techniques and advanced pattern recognition approaches, possibly by incorporating machine learning, to analyze all this data.

\subsection{Future Steps}

Many of the issues that result in more of a semiautomated approach are technical factors in the OCT system which make the preprocessing step necessary, such as the speckle noise and image artifacts that appear in the resulting OCT images. Current research efforts at our institution have largely eliminated these factors, and future OCT image analysis could potentially eliminate all preprocessing steps and become a fully automated process. We are also designing additional algorithms to automatically both segment airway wall layers more efficiently and analyze tissue composition, such as three-dimensional tissue segmentation. ${ }^{50}$ This would simplify the process of recognizing upper airway tissue morphology changes and dramatically speed up diagnosis of upper airway diseases. We expect that our work will be reproducible and of benefit to other OCT imaging teams. With minimal in-person training, we foresee other medical centers being able to utilize our segmentation algorithms. Moreover, we believe that with minimal alterations, if any, our algorithm will be translated to measure other tissue types.

The integration of vertical-cavity surface-emitting lasers (VCSEL) technology with OCT systems is a rapidly developing arena, as these imaging systems feature vast improvements in image quality with refined optics and probe design. ${ }^{51}$ VCSEL OCT imaging is an active area of interest in our group, and we aim to perform further imaging in intubated neonates and gain improved quality image sets to better understand relationships between OCT data and clinical parameters.

\section{Conclusion}

OCT is a high-resolution diagnostic imaging modality that has tremendous potential as a means to objectively and serially analyze the airway in intubated neonates. The automated image analysis algorithm described in this report offers an efficient and precise solution to analyzing large-volume OCT data stacks. With automated image analysis, OCT may ultimately offer clinicians real-time information about the health of the intubated neonatal airway and aid in airway management.

\section{Disclosures}

The author(s) declared no potential conflicts of interest with respect to the research, authorship, and/or publication of this article. 


\section{Acknowledgments}

We would like to thank Kathryn Osann, $\mathrm{PhD}$, for her help with the statistical analysis. The author(s) disclosed receipt of the following financial support for the research, authorship, and/or publication of this article: the Air Force Office of Scientific Research (FA9550-04-1-0101), the National Institutes of Health (NIH/NHLBI/NIDCD 1-R01-HL103764-01 and NIH/NHLBI 1-R01-HL105215-01), Tobacco-Related Disease Research Program (TRDRP 19KT-0034), the Leading Foreign Research Institute Recruitment Program through the National Research Foundation of Korea (NRF) funded by the Ministry of Science and ICT (MSIT) (NRF-2018K1A4A3A02060572).

\section{References}

1. H. Suzumura et al., "Role of infection in the development of acquired subglottic stenosis in neonates with prolonged intubation," Pediatr. Int. 42(5), 508-513 (2000).

2. D. L. Walner, M. S. Loewen, and R. E. Kimura, "Neonatal subglottic stenosis-incidence and trends," Laryngoscope 111(1), 48-51 (2001).

3. R. E. Thomas et al., "Severe acquired subglottic stenosis in neonatal intensive care graduates: a case-control study," Arch. Dis. Childhood Fetal Neonatal Ed. 103(4), F349-F354 (2018).

4. K. Arianpour et al., "Pediatric acquired subglottic stenosis: associated costs and comorbidities of 7,981 hospitalizations," Int. J. Pediatr. Otorhinolaryngol. 117, 51-56 (2019).

5. A. J. Feinstein et al., "Endoscopic management of subglottic stenosis," JAMA Otolaryngol. Neck Surg. 143(5), 500-505 (2017).

6. V. Volgger et al., "Long-range Fourier domain optical coherence tomography of the pediatric subglottis," Int. J. Pediatr. Otorhinolaryngol. 79(2), 119-126 (2015).

7. O. Ajose-Popoola et al., "Diagnosis of subglottic stenosis in a rabbit model using long-range optical coherence tomography," Laryngoscope 127(1), 64-69 (2017).

8. B. J. F. Wong et al., "In vivo optical coherence tomography of the human larynx: normative and benign pathology in 82 patients," Laryngoscope 115(11), 1904-1911 (2005).

9. G. K. Sharma et al., "Quantitative evaluation of adult subglottic stenosis using intraoperative long-range optical coherence tomography," Ann. Otol. Rhinol. Laryngol. 125(10), 815-822 (2016).

10. J. L. Lin et al., "Real-time subglottic stenosis imaging using optical coherence tomography in the rabbit," JAMA Otolaryngol. Head Neck Surg. 139(5), 502-509 (2013).

11. T. Klein and R. Huber, "High-speed OCT light sources and systems [Invited]," Biomed. Opt Express 8(2), 828-859 (2017).

12. J. F. de Boer, R. Leitgeb, and M. Wojtkowski, "Twenty-five years of optical coherence tomography: the paradigm shift in sensitivity and speed provided by Fourier domain OCT [Invited]," Biomed. Opt. Express 8(7), 3248-3280 (2017).

13. G. J. Ughi et al., "Automated tissue characterization of in vivo atherosclerotic plaques by intravascular optical coherence tomography images," Biomed. Opt. Express 4(7), 1014-1030 (2013).

14. S. Buoro et al., "Evaluation of nucleated red blood cell count by Sysmex XE-2100 in patients with thalassaemia or sickle cell anaemia and in neonates," Blood Transfus. 13(4), 588-594 (2015).

15. E. Bengtsson and P. Malm, "Screening for cervical cancer using automated analysis of PAP-smears," Comput. Math. Methods Med. 2014, 1-12 (2014).

16. P. Lambin et al., "Radiomics: extracting more information from medical images using advanced feature analysis," Eur. J. Cancer 48(4), 441-446 (2012).

17. G. Castellano et al., "Texture analysis of medical images," Clin. Radiol. 59(12), 1061-1069 (2004).

18. K. W. Gossage et al., "Texture analysis of optical coherence tomography images: feasibility for tissue classification," J. Biomed. Opt. 8(9), 570-575 (2003).

19. G. K. Sharma et al., "Long-range optical coherence tomography of the neonatal upper airway for early diagnosis of intubation-related subglottic injury," Am. J. Respir. Crit. Care Med. 192(12), 1504-1513 (2015).
20. J. Jing et al., "High-speed upper-airway imaging using full-range optical coherence tomography," J. Biomed. Opt. 17(11), 110507 (2012).

21. J. J. Armstrong et al., "Quantitative upper airway imaging with anatomic optical coherence tomography," Am. J. Respir. Crit. Care Med. 173(2), 226-233 (2006).

22. C. A. Toth et al., "A comparison of retinal morphology viewed by optical coherence tomography and by light microscopy," Arch. Ophthalmol. 115(11), 1425-1428 (1997).

23. L. Qi et al., "Automatic airway wall segmentation and thickness measurement for long-range optical coherence tomography images," Opt Express 23(26), 033992 (2015).

24. Z. Jian et al., "Three-dimensional speckle suppression in optical coherence tomography based on the curvelet transform," Opt Express 18(2), 1024-1032 (2010).

25. Y. Du et al., "Speckle reduction in optical coherence tomography images based on wave atoms," J. Biomed. Opt. 19(5), 056009 (2014).

26. J. Zhang, J. S. Nelson, and Z. Chen, "Removal of a mirror image and enhancement of the signal-to-noise ratio in Fourier-domain optical coherence tomography using an electro-optic phase modulator," Opt. Lett. 30(2), 147-149 (2005).

27. S. Adabi et al., "Universal in vivo textural model for human skin based on optical coherence tomograms," Sci. Rep. 7, 17912 (2017).

28. D. C. Adams et al., "Automated segmentation and quantification of airway mucus with endobronchial optical coherence tomography," Biomed. Opt Express 8(10), 4729-4741 (2017).

29. M. R. Hee et al., "Optical coherence tomography of the human retina," Arch. Ophthalmol. 113(9), 325-332 (1995).

30. I. K. Jang et al., "Visualization of coronary atherosclerotic plaques in patients using optical coherence tomography: comparison with intravascular ultrasound," J. Am. Coll. Cardiol. 39(4), 604-609 (2002).

31. N. D. Gladkova et al., "In vivo optical coherence tomography imaging of human skin: norm and pathology," Skin Res. Technol. 6(1), 6-16 (2000).

32. A. M. D. Lee et al., "Validation of airway wall measurements by optical coherence tomography in porcine airways," PLoS One 9(6), e100145 (2014).

33. Y. Chen et al., "Validation of human small airway measurements using endobronchial optical coherence tomography," Respir. Med. 109(11), 1446-1453 (2015).

34. M. Kirby et al., "Automated segmentation of porcine airway wall layers using optical coherence tomography: comparison with manual segmentation and histology," Proc. SPIE 8927, 89271D (2014).

35. D. C. Fernandez, H. M. Salinas, and C. A. Puliafito, "Automated detection of retinal layer structures on optical coherence tomography images," Opt. Express 13(25), 10200-10216 (2005).

36. W. Shalish et al., "Predictors of extubation readiness in preterm infants: a systematic review and meta-analysis," Arch. Dis. Childhood Fetal Neonatal Ed. 104(1), F89-F97 (2019).

37. J. P. Lerch et al., "Automated cortical thickness measurements from MRI can accurately separate Alzheimer's patients from normal elderly controls," Neurobiol. Aging 29(1), 23-30 (2008).

38. K. Van Leemput et al., "Automated segmentation of hippocampal subfields from ultra-high resolution in vivo MRI," Hippocampus 19(6), 549-557 (2009).

39. S. G. Armato and W. F. Sensakovic, "Automated lung segmentation for thoracic CT: impact on computer-aided diagnosis," Acad. Radiol. 11(9), 1011-1021 (2004).

40. E. A. Hoffman et al., "Characterization of the interstitial lung diseases via density-based and texture-based analysis of computed tomography images of lung structure and function," Acad. Radiol. 10(10), 1104 1118 (2003).

41. H. O. Coxson and S. Lam, "Quantitative assessment of the airway wall using computed tomography and optical coherence tomography," Proc. Am. Thorac. Soc. 6(5), 439-443 (2009).

42. J. Cheng et al., "Correction: enhanced performance of brain tumor classification via tumor region augmentation and partition," PLoS One 10(12), e0144479 (2015).

43. H. Liu et al., "Cirrhosis classification based on texture classification of random features," Comput. Math. Methods Med. 2014, 1-8 (2014).

44. L. B. Mostaço-Guidolin et al., "Collagen morphology and texture analysis: from statistics to classification," Sci. Rep. 3(1), 2190 (2013). 
45. M. Gloesmann et al., "Histologic correlation of pig retina radial stratification with ultrahigh-resolution optical coherence tomography," Invest. Ophthalmol. Vis. Sci. 44(4), 1696-1703 (2003).

46. E. Scalco and G. Rizzo, "Texture analysis of medical images for radiotherapy applications," Br. J. Radiol. 90(1070), 20160642 (2016)

47. W. Pedrycz, "Fuzzy sets in pattern recognition: methodology and methods," Pattern Recognit. 23(1-2), 121-146 (1990).

48. M. A. Mayer et al., "Fuzzy C-means clustering for retinal layer segmentation on high resolution OCT images," in 19th Biosignal Conf. (2008).
49. J. Malek et al., "Automated breast cancer diagnosis based on GVFsnake segmentation, wavelet features extraction and fuzzy classification," J. Signal Process. Syst. 55(1-3), 49-66 (2009).

50. L. Qi et al., "Automatic three-dimensional segmentation of endoscopic airway OCT images," Biomed. Opt. Express 10(2), 642-656 (2019).

51. I. Grulkowski et al., "Retinal, anterior segment and full eye imaging using ultrahigh speed swept source OCT with vertical-cavity surface emitting lasers," Biomed. Opt. Express 3(11), 2733-2751 (2012).

Biographies of the authors are not available. 